\title{
The clinical course of low back pain: a meta-analysis comparing outcomes in randomised clinical trials (RCTs) and observational studies
}

\author{
Majid Artus*, Danielle van der Windt, Kelvin P Jordan and Peter R Croft
}

\begin{abstract}
Background: Evidence suggests that the course of low back pain (LBP) symptoms in randomised clinical trials (RCTs) follows a pattern of large improvement regardless of the type of treatment. A similar pattern was independently observed in observational studies. However, there is an assumption that the clinical course of symptoms is particularly influenced in RCTs by mere participation in the trials. To test this assumption, the aim of our study was to compare the course of LBP in RCTs and observational studies.

Methods: Source of studies CENTRAL database for RCTs and MEDLINE, CINAHL, EMBASE and hand search of systematic reviews for cohort studies. Studies include individuals aged 18 or over, and concern non-specific LBP. Trials had to concern primary care treatments. Data were extracted on pain intensity. Meta-regression analysis was used to compare the pooled within-group change in pain in RCTs with that in cohort studies calculated as the standardised mean change (SMC).

Results: 70 RCTs and 19 cohort studies were included, out of 1134 and 653 identified respectively. LBP symptoms followed a similar course in RCTs and cohort studies: a rapid improvement in the first 6 weeks followed by a smaller further improvement until 52 weeks. There was no statistically significant difference in pooled SMC between RCTs and cohort studies at any time point:- 6 weeks: RCTs: SMC 1.0 (95\% Cl 0.9 to 1.0) and cohorts 1.2 (0.7to 1.7); 13 weeks: RCTs 1.2 (1.1 to 1.3) and cohorts 1.0 (0.8 to 1.3); 27 weeks: RCTs 1.1 (1.0 to 1.2) and cohorts 1.2 (0.8 to 1.7); 52 weeks: RCTs 0.9 (0.8 to 1.0) and cohorts 1.1 (0.8 to 1.6).

Conclusions: The clinical course of LBP symptoms followed a pattern that was similar in RCTs and cohort observational studies. In addition to a shared 'natural history', enrolment of LBP patients in clinical studies is likely to provoke responses that reflect the nonspecific effects of seeking and receiving care, independent of the study design.
\end{abstract}

\section{Background}

Well-conducted randomised clinical trials (RCTs) generally provide the strongest evidence for the effectiveness of treatments. RCTs on the effectiveness of treatments for non-specific low back pain have not found evidence for a clear superiority of any treatment [1-3]. Yet, low back pain symptoms tend to improve in RCTs regardless of the treatment provided. Such improvement seems to follow a pattern common to all treatment arms, of rapid early improvement within the first 6 weeks reaching a plateau over the following 12 months [4]. This is explained at least partly by the 'natural history' (i.e. the

* Correspondence: m.artus@keele.ac.uk

Arthritis Research UK Primary Care Centre, Primary Care Sciences, Keele University, Keele, Staffordshire ST5 5BG, UK propensity for symptoms to improve without treatment). With the use of treatment this is referred to as the 'clinical course' of symptoms. The clinical course of back pain has been assessed in observational (cohort) studies $[5,6]$. It was also found to follow a pattern of general improvement that starts rapidly and plateaus over time. Although this suggests a similarity between RCTs and cohort studies, there is no clear evidence for this from direct comparison. More importantly, it is not clear whether the size of overall symptom improvement is the same in these two groups of studies. There is only a limited evidence for a direct comparison, mainly comparing RCTs with non-randomised trials and observational studies that included comparator groups [7].

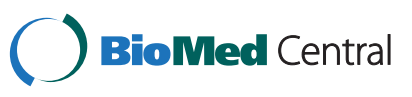


There is an assumption that the course of symptoms in RCTs is different from that in cohort studies. It has been suggested that the mere participation in a trial influences the course of symptoms $[8,9]$. This might be explained by benefits perceived by participants and assumed to be related to the intensive assessment and monitoring. The so called 'Hawthorne effect' was quoted as an example of how individuals change behaviour due to the attention they receive from researchers. [10-12]. Although this is expected to apply to all studies, it might be relatively more pronounced in RCTs compared with cohort studies.

Another issue is whether participants in RCTs are in some way different from the average person presenting for care in usual clinical practice. Whether their willingness to be randomly allocated to a treatment or a placebo makes these individuals different from the average patient to whom the results of RCTs will be applied. If true, this raises the issue of whether participants in RCTs are less representative of the average patients compared with participants in observational studies in which patients are not randomised.

It is therefore important to establish the evidence for the similarity or otherwise, in the pattern and the size of back pain symptom improvement in these two types of studies. This would test the assumption that mere willingness to enrol in RCTs and be randomised to treatments would influence the clinical course of symptoms. This would have potentially important implications on interpreting the results of RCTs and their generalizability in clinical practice.

The aim of this systematic review and meta-analysis was to compare changes in low back pain symptoms over time in RCT participants with those of participants in observational cohort studies.

\section{Methods \\ Criteria for inclusion}

Included were studies (RCTs and prospective observational cohort studies) conducted for primary care treatment for LBP (e.g. analgesia, exercises, manipulation therapy) among individuals aged 18 or over. Studies had to provide baseline and follow-up data on the designated primary outcome measure of pain intensity, measured on a Numerical Rating Scale (NRS) or Visual Analogue Scale (VAS). Only studies published in English were included. Also excluded were studies conducted among patients with specific LBP (e.g. cancer or inflammatory arthritis), post-operative or post-traumatic back pain, or back pain associated with pregnancy or labour.

\section{Searching and selection of studies}

To meet the specific aims of the study, the literature search did not have to be exhaustive, but to provide sufficiently large pool of studies. The Cochrane Central Register of Controlled Trials (CENTRAL) was therefore chosen as a sufficient data source for RCTs.. This search was an update (up to April 2012) of a strategy previously used and described elsewhere [4]. For observational studies, a literature search was conducted for the same time period using the databases of AMED, EMBASE, MEDLINE and CINAHL based on the keywords 'low back pain', 'back pain', 'spinal pain', 'primary care', 'general practice', 'population', 'cohort', 'observational', 'prognosis', predictor' and 'course'. The detailed search strategy is shown in Additional file 1. References accompanying relevant systematic reviews and included cohort studies were also hand-checked to identify additional eligible studies.

The literature search was conducted by MA and screening of citations/abstracts ad selection of RCTs and cohort studies applying the inclusion criteria was conducted by MA, DVdW \& KPJ.

\section{Data extraction}

The extracted data included:

1. Study characteristics (publication year, country of study, clinical setting, study design, sample size).

2. Participants' characteristics (mean age;\% female; duration of symptoms).

3. Interventions: name, dose and provider.

4. Outcome: baseline and follow up mean scores (and baseline standard deviation (SD)) for pain intensity.

\section{Analysis}

Firstly, RCTs as a single group were compared with observational studies. Secondly, RCTs were sub-grouped into efficacy and pragmatic trials, based on whether the trial included a placebo, sham or no treatment, with such trials being grouped as efficacy trials. RCTs that included comparator treatment of usual care or waiting list arms were classified as pragmatic trials. To compare studies groups that are similar with regard to the type of treatment, a separate analysis was conducted to compare cohort studies with RCT arms that received 'usual care'. Each RCT sub-group was compared separately with observational studies.

Pain intensity scores were converted to a zero to 100 scale (least to most severe) where necessary by multiplication. Meta-analysis using a random effects model was performed using STATA/IC 11 software to compute pooled mean pain intensity scores (and 95\% confidence intervals) at baseline and follow up, separately for RCT treatment arms and for observational studies. Commonly used followup times of 6, 13, 27 and 52 weeks were selected for comparison. Data on other time points were considered to fall 
within the selected points if they were within a threeweek range.

To compare the size of improvement in outcome scores in RCTs and observational studies, the standardized mean change (SMC) [13] was calculated for each RCT treatment arm and observational study by subtracting the follow-up mean outcome score from the baseline mean score and dividing by the standard deviation (SD) of baseline scores. Pooled SMCs were calculated using random effects meta-analysis. SMCs over 0.8 were considered large, $0.5-0.8$ moderate and less than 0.5 small [14]. The 95\% Confidence Intervals for SMCs were calculated using the formula described by Hozo et al. [15]. The variance (squared standard deviation, $\sigma 2$ ) of response size was calculated using the following formula [15]:

$$
\sigma 2=2(1-\rho) / n[(n-1) /(n-3)][1+n / 2(1-\rho) \delta 2]-\delta 2 /[c(n-1)] 2
$$

Where: c (n-1) approximates 1 - [3/ 4(n-1) -1], $\rho$ is the population correlation between baseline and followup scores which was estimated as $0.5, \mathrm{n}$ is sample size and $\delta$ is the SMC. Heterogeneity of studies' estimates was assessed by computing $I^{2}$ statistic [16], where zero indicates no variation between studies and 100\% indicates that all variation is the result of variation between studies. Meta-regression analyses were conducted to test the significance of the difference in the size of SMCs between RCTs and observational studies at the selected follow up points.

\section{Results}

\section{Included studies}

The updated search for RCTs yielded a total of 1134 citations of which papers for 70 RCTs (165 treatment arms) satisfied the inclusion criteria and provided pain intensity data useful for analysis (Figure 1). The search for observational studies yielded a total of 653 citations (Figure 2), and data for pain intensity useful for analysis were provided in 15 papers. Relevant data were obtained for further four papers by contacting authors, allowing analysis of pain intensity data from papers for a total of 19 observational studies.

\section{Characteristics of study setting and population}

A list of the included RCTs and observational studies and their population characteristics are presented in Tables 1 \& 2. They were conducted in more than 13 countries including the USA, Australia, and European countries during a period spanning two decades. They are comparable in terms of age distribution, gender composition and mean baseline pain intensity (Table 3 ). It appears that compared with observational studies, RCTs included a larger percentage of participants described as having chronic low back pain (57\% in RCTs vs $11 \%$ in cohorts). However, these figures need to be interpreted with caution as observational studies often included a mixture of patients with acute and chronic back pain (19\% in RCTs vs $63 \%$ in cohorts).

The setting of RCTs included general practice (18 RCTs), occupational health care departments (15 RCTs)

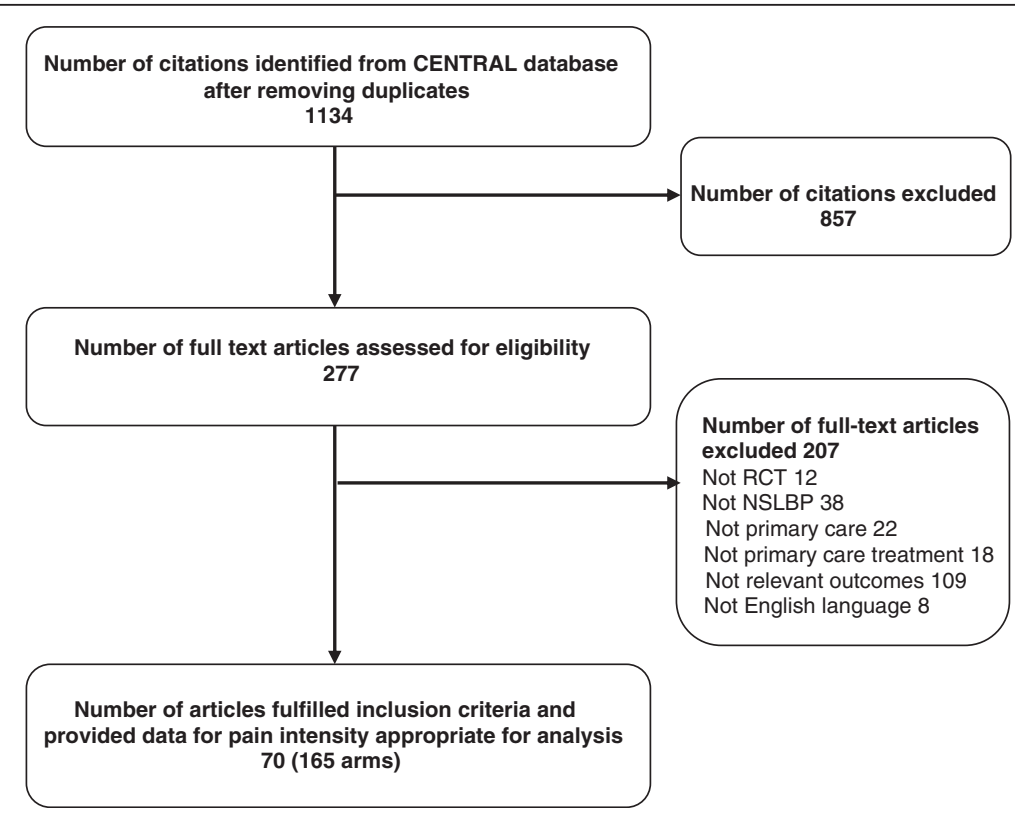

Figure 1 Identification and inclusion of RCTs in the systematic review. 


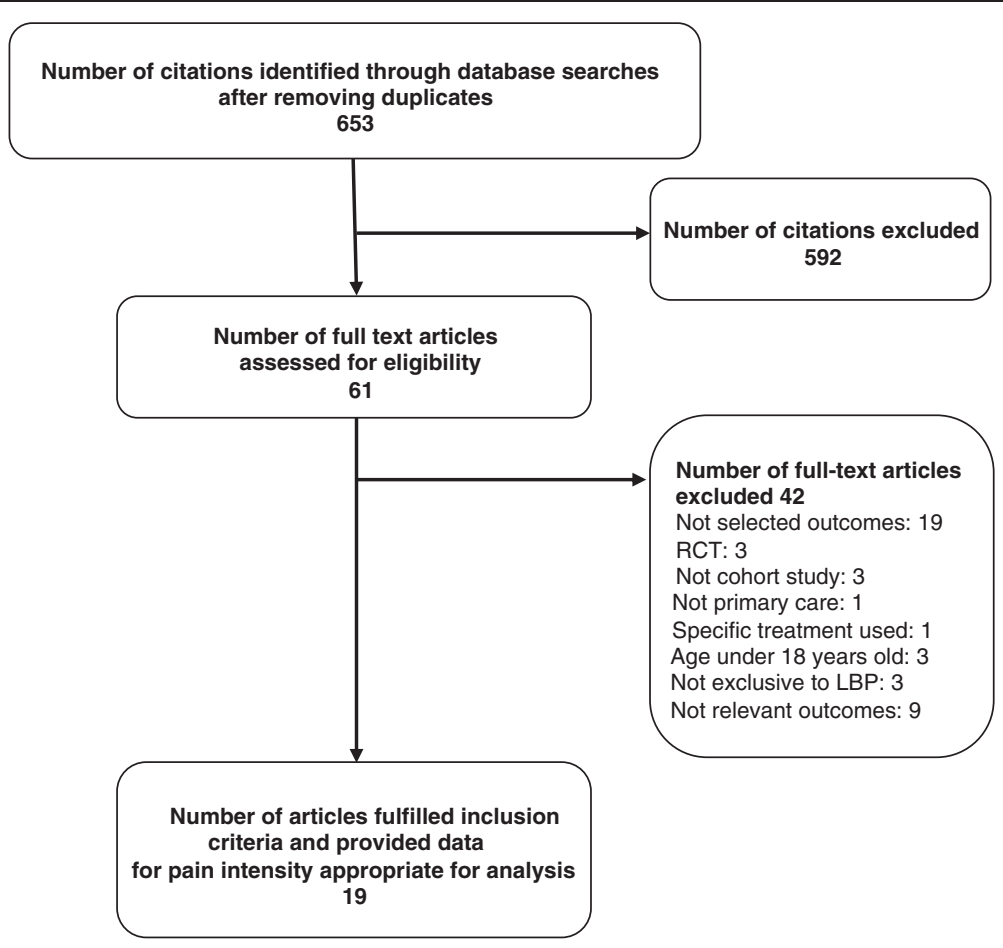

Figure 2 Identification and inclusion of observational cohort studies in the systematic review.

Table 1 Characteristics of included observational cohort studies (n 19)

\begin{tabular}{|c|c|c|c|c|c|}
\hline Author and country & Population and setting & Age, mean (y) & Female $\%$ & Type of back pain & Sample size \\
\hline Bakker et al., Netherlands [17] & GP consulters & 41 & 48 & Acute & 97 \\
\hline Bekkering et al., Netherlands [18] & Physiotherapy consulters & 45 & 52 & Mixed & 500 \\
\hline Carey et al., USA [19] & GP and chiropractic consulters & 42 & 52 & Acute & 1628 \\
\hline Chenot et al., Germany [20] & GP consulters & 44 & & Mixed & 1342 \\
\hline Coste et al., France [21] & GP consulters & 46 & 40 & Acute & 103 \\
\hline Demmelmeir et al., Sweden [22] & General population & 42 & 55 & Mixed & 379 \\
\hline Dunn et al., UK [23] & GP consulters & & & Mixed & 206 \\
\hline Grotle et al., Norway [24] & Primary care & 38 & 55 & Acute & 123 \\
\hline Hass et al., Netherlands [25] & Community chiropractic clinics & 43 & 53 & Mixed & 2780 \\
\hline Kovacs et al., Spain [26] & GP consulters & 46 & 52 & Mixed & 648 \\
\hline McGuirk et al., Australia [27] & GP consulters & 53 & 57 & Acute & 83 \\
\hline Miller et al., UK [28] & GP consulters & 39 & 60 & Mixed & 211 \\
\hline Nyiendo et al., USA [29] & Medical and chiropractic clinics & & & Chronic & 835 \\
\hline Perreault et al., Canada [30] & Physiotherapy departments & 51 & & Mixed & 78 \\
\hline Sefarlis et al., Sweden [31] & GP consulters & 39 & & Acute & 60 \\
\hline Sharma et al., USA [32] & Medical and chiropractic clinics consulters & 40 & 50 & Mixed & 2872 \\
\hline Tamcan et al., Switzerland [33] & General population & 42 & 50 & Chronic & 340 \\
\hline van Hoogan et al., Netherlands [34] & GP consulters & 44 & 55 & Mixed & 443 \\
\hline van Tulder et al., Netherlands [35] & GP consulters & 41 & 49 & Mixed & 368 \\
\hline
\end{tabular}


Table 2 Characteristics of included RCTs (n 70)

\begin{tabular}{|c|c|c|c|c|c|c|}
\hline Author and country & Setting & Treatment & $\begin{array}{l}\text { Age, } \\
\text { mean } \\
(y)\end{array}$ & $\begin{array}{c}\text { Female } \\
(\%)\end{array}$ & $\begin{array}{c}\text { Duration of } \\
\text { back pain, } \\
\text { mean (weeks) }\end{array}$ & $\begin{array}{l}\text { Sample } \\
\text { size of } \\
\text { trial arms }\end{array}$ \\
\hline \multirow{3}{*}{$\begin{array}{l}\text { Albaladejo et al., } \\
\text { Spain [36] }\end{array}$} & Primary care & Education \& physiotherapy & 51 & 68 & & 100 \\
\hline & & Education & 51 & 63 & & 139 \\
\hline & & Usual GP care & 53 & 72 & & 109 \\
\hline \multirow{2}{*}{$\begin{array}{l}\text { Arribas et al., } \\
\text { Spain [37] }\end{array}$} & National health centres & GDS physical therapy & 39 & 64 & & 78 \\
\hline & & Electrotherapy & 39 & 64 & & 67 \\
\hline \multirow{2}{*}{$\begin{array}{l}\text { Bendix et al., } \\
\text { Denmark [38] }\end{array}$} & General practice & Functional restoration (PT + OT + Psychological) & 40 & 66 & & 48 \\
\hline & & $\begin{array}{l}\text { Outpatient intensive physical training: } \\
\text { Aerobics + strengthening exercises + } \\
\text { fitness machines }\end{array}$ & 43 & 69 & & 51 \\
\hline \multirow[t]{3}{*}{$\begin{array}{l}\text { Bronfort et al., } \\
\text { USA [39] }\end{array}$} & College outpatient clinic & $\begin{array}{l}\text { Spinal manipulation \& trunk strengthening } \\
\text { exercise }\end{array}$ & 41 & 54 & 156 & 71 \\
\hline & & NSAID \& Trunk strengthening exercise & 40 & 44 & 104 & 52 \\
\hline & & Spinal manipulation \& Stretching exercise & 41 & 39 & 120 & 51 \\
\hline \multirow{3}{*}{$\begin{array}{l}\text { Bronfort et al., } \\
\text { USA [40] }\end{array}$} & Physical therapy clinic & Supervised exercises & 45 & 57 & 249 & 100 \\
\hline & & Chiropractic & 45 & 66 & 250 & 100 \\
\hline & & Home exercises & 46 & 58 & 250 & 101 \\
\hline \multirow{2}{*}{$\begin{array}{l}\text { Browder et al., } \\
\text { USA [41] }\end{array}$} & Physical therapy clinics & Extension orientated exercises & 40 & 31 & 9 & 26 \\
\hline & & Strengthening exercises & 38 & 32 & 9 & 22 \\
\hline \multirow[t]{2}{*}{$\begin{array}{l}\text { Burton et al., } \\
\text { UK [42] }\end{array}$} & General practice & $\begin{array}{l}\text { The Back Book + usual care } \\
\text { (GP \& osteopathic care) }\end{array}$ & & 11 & & 83 \\
\hline & & $\begin{array}{l}\text { The traditional Handy Hints \& usual care } \\
\text { (GP \& osteopathic care) }\end{array}$ & & 12 & & 79 \\
\hline \multirow[t]{2}{*}{$\begin{array}{l}\text { Cambron et al., } \\
\text { USA [43] }\end{array}$} & $\begin{array}{l}\text { Chiropractic clinic }+ \\
\text { hospital clinic }+ \text { General } \\
\text { population }\end{array}$ & Chiropractic flexion distraction procedure & 42 & 34 & & 123 \\
\hline & & Active trunk exercise program & 41 & 41 & & 112 \\
\hline \multirow{3}{*}{$\begin{array}{l}\text { Cecchi et al., } \\
\text { Italy [44] }\end{array}$} & Rehabilitation department & Spinal manipulation & 58 & 69 & & 70 \\
\hline & & Individual physiotherapy & 61 & 61 & & 70 \\
\hline & & Back school & 58 & 70 & & 70 \\
\hline \multirow{2}{*}{$\begin{array}{l}\text { Chan et al., } \\
\text { Hong Kong [45] }\end{array}$} & Physiotherapy & Aerobic training & 47 & 79 & 54 & 24 \\
\hline & & Usual physiotherapy & 46 & 77 & 63 & 22 \\
\hline \multirow{2}{*}{$\begin{array}{l}\text { Chang et al., } \\
\text { Taiwan [46] }\end{array}$} & General population & Piroxicam sachet & 34 & 30 & & 23 \\
\hline & & Piroxicam tablets & 34 & 26 & & 19 \\
\hline \multirow[t]{2}{*}{$\begin{array}{l}\text { Chok et al., } \\
\text { Singapore [47] }\end{array}$} & $\begin{array}{l}\text { Physiotherapy + } \\
\text { Orthopaedic clinics + A/E }\end{array}$ & $\begin{array}{l}\text { Physical therapy (endurance exercise } \\
\text { at the PT department) + back hot pack }\end{array}$ & 38 & 20 & 4 & 38 \\
\hline & & Back hot pack (Home) & 34 & 29 & 4 & 28 \\
\hline \multirow{2}{*}{$\begin{array}{l}\text { Costa et al., } \\
\text { Australia [48] }\end{array}$} & Physical therapy clinics & Exercise & 55 & 58 & 335 & 77 \\
\hline & & Detuned diathermy and detuned USS & 53 & 62 & 328 & 77 \\
\hline Constant et al., & General practice & Spa therapy \& usual GP care & & & & 63 \\
\hline
\end{tabular}


Table 2 Characteristics of included RCTs (n 70) (Continued)

\begin{tabular}{|c|c|c|c|c|c|c|}
\hline & & Waiting list group \& usual GP care & & & & 63 \\
\hline \multirow{3}{*}{$\begin{array}{l}\text { Critchley et al., } \\
\text { UK [50] }\end{array}$} & Physiotherapy department & Individual physiotherapy & 45 & 59 & 275 & 71 \\
\hline & & Spinal stabilisation & 44 & 71 & 346 & 72 \\
\hline & & Pain management & 44 & 62 & 348 & 69 \\
\hline \multirow{2}{*}{$\begin{array}{l}\text { Di Cesare et al., } \\
\text { Italy [51] }\end{array}$} & Physical therapy clinics & Trigger point mesotherapy & 53 & 55 & 22 & 29 \\
\hline & & Acupuncture point mesotherapy & 53 & 55 & 21 & 33 \\
\hline \multirow{3}{*}{$\begin{array}{l}\text { Djavid et al., } \\
\text { Iran [52] }\end{array}$} & Occupational clinic & Low level laser (LLL) & 40 & 56 & 118 & 20 \\
\hline & & LLL + exercise & 38 & 37 & 110 & 21 \\
\hline & & Placebo LLL + exercise & 36 & 17 & 106 & 20 \\
\hline \multirow{2}{*}{$\begin{array}{l}\text { Dufour et al., } \\
\text { Denmark [53] }\end{array}$} & Rheumatology clinics & Group based multidisciplinary therapy & 41 & 57 & 514 & 142 \\
\hline & & Individual therapist assisted exercises & 41 & 56 & 540 & 144 \\
\hline \multirow{2}{*}{$\begin{array}{l}\text { Dundar et al., } \\
\text { Turkey [54] }\end{array}$} & Physical therapy clinics & Aquatic exercise & 35 & 47 & & 32 \\
\hline & & Land based exercise & 35 & 48 & & 33 \\
\hline \multirow{2}{*}{$\begin{array}{l}\text { Fritz et al., } \\
\text { USA [55] }\end{array}$} & Physical therapy clinics & Traction plus EOT & 42 & 55 & & 31 \\
\hline & & Extension orientated therapy (EOT) & 41 & 58 & & 33 \\
\hline \multirow{2}{*}{$\begin{array}{l}\text { Frost et al., } \\
\text { UK [56] }\end{array}$} & Physiotherapy & Routine physiotherapy \& advice book & 42 & 58 & & 144 \\
\hline & & Advice from physiotherapist \& advice book & 40 & 47 & & 142 \\
\hline \multirow[t]{4}{*}{$\begin{array}{l}\text { Geisser et al., } \\
\text { USA [57] }\end{array}$} & $\begin{array}{l}\text { University spinal } \\
\text { programme }\end{array}$ & $\begin{array}{l}\text { Manual therapy \& Specific exercise } \\
\text { (self corrections, stretching, strengthening) }\end{array}$ & 39 & 67 & 284 & 26 \\
\hline & & Sham Manual therapy \& Specific exercise & 39 & 56 & 370 & 25 \\
\hline & & Manual Therapy \& Non-specific exercise & 37 & 80 & 370 & 24 \\
\hline & & Sham Manual Therapy \& non-specific exercise & 46 & 61 & 284 & 25 \\
\hline \multirow{2}{*}{$\begin{array}{l}\text { George et al., } \\
\text { USA [58] }\end{array}$} & Physical therapy & Standard care physical therapy & 37 & 53 & 4 & 32 \\
\hline & & Fear-avoidance based physical therapy & 40 & 62 & 4 & 34 \\
\hline \multirow{2}{*}{$\begin{array}{l}\text { Glasov et al., } \\
\text { Australia [59] }\end{array}$} & General population & Laser acupuncture & 58 & 95 & & 45 \\
\hline & & Sham laser & 49 & 62 & & 45 \\
\hline \multirow[t]{2}{*}{$\begin{array}{l}\text { Glomsrod et al., } \\
\text { Norway }[60]\end{array}$} & $\begin{array}{l}\text { Physicians clinics and } \\
\text { General population }\end{array}$ & $\begin{array}{l}\text { Active back school (Lectures and } \\
\text { back exercises) }\end{array}$ & 41 & 65 & & 37 \\
\hline & & Usual medical care & 39 & 57 & & 35 \\
\hline \multirow[t]{3}{*}{$\begin{array}{l}\text { Goldby et al., } \\
\text { UK [61] }\end{array}$} & $\begin{array}{l}\text { General practice + hospital } \\
\text { physicians }\end{array}$ & $\begin{array}{l}\text { Spinal stabilisation \& Attending } \\
\text { the back school }\end{array}$ & 43 & 68 & & 84 \\
\hline & & $\begin{array}{l}\text { Manual therapy \& Attending the } \\
\text { back school }\end{array}$ & 41 & 70 & & 89 \\
\hline & & $\begin{array}{l}\text { Education (Booklet: Back in action) \& } \\
\text { Attending the back school }\end{array}$ & 42 & 68 & & 40 \\
\hline $\begin{array}{l}\text { Hay et al., } \\
\text { UK [62] }\end{array}$ & General practice & $\begin{array}{l}\text { A brief programme of pain management } \\
\text { (general fitness and exercise at clinic and home, } \\
\text { explanation about pain mechanisms, distress, } \\
\text { encouragement of positive coping strategies, } \\
\text { overcoming fear of "hurt = harm", and } \\
\text { implementation of a graded return } \\
\text { to usual activities) }\end{array}$ & 40 & 50 & & 201 \\
\hline
\end{tabular}


Table 2 Characteristics of included RCTs (n 70) (Continued)

\begin{tabular}{|c|c|c|c|c|c|c|}
\hline & & $\begin{array}{l}\text { Physiotherapy including manual therapy } \\
\text { techniques }\end{array}$ & 41 & 55 & & 201 \\
\hline \multirow{3}{*}{$\begin{array}{l}\text { Heymans et al., } \\
\text { Netherlands [63] }\end{array}$} & Occupational healthcare & Usual Dutch occupational physician care & 41 & 17 & 35 & 103 \\
\hline & & Low intensity back school & 41 & 22 & 35 & 98 \\
\hline & & High intensity back school & 40 & 23 & 35 & 98 \\
\hline \multirow{4}{*}{$\begin{array}{l}\text { Hseih et al., } \\
\text { USA [64] }\end{array}$} & General population & Joint manipulation \& myofascial therapy & 48 & 33 & 12 & 52 \\
\hline & & Joint manipulation & 47 & 33 & 12 & 48 \\
\hline & & Myofascial therapy & 49 & 33 & 12 & 51 \\
\hline & & Back school & 48 & 40 & 11 & 48 \\
\hline \multirow[t]{3}{*}{$\begin{array}{l}\text { Hurley et al., } \\
\text { UK [65] }\end{array}$} & $\begin{array}{l}\text { Physiotherapy + General } \\
\text { practice + self referral }\end{array}$ & $\begin{array}{l}\text { Manipulation therapy (Passively move } \\
\text { intervertebral joint within or beyond its range) }\end{array}$ & 40 & 57 & 8 & 80 \\
\hline & & Interferential therapy (Electrical stimulation) & 40 & 62 & 8 & 80 \\
\hline & & Manipulation \& interferential therapy & 41 & 60 & 8 & 80 \\
\hline \multirow{4}{*}{$\begin{array}{l}\text { Hurwitz et al., } \\
\text { USA [66] }\end{array}$} & Managed care facility & Chiropractic care only & 52 & 49 & & 169 \\
\hline & & $\begin{array}{l}\text { Chiropractic care \& physical modalities } \\
\text { (Heat/cold, USS) }\end{array}$ & 54 & 58 & & 172 \\
\hline & & $\begin{array}{l}\text { Medical care (excluding physical treatment) } \\
\text { only }\end{array}$ & 49 & 47 & & 170 \\
\hline & & $\begin{array}{l}\text { Medical care \& physical modalities } \\
\text { (Heat/cold, USS) }\end{array}$ & 49 & 54 & & 170 \\
\hline \multirow{2}{*}{$\begin{array}{l}\text { Hurwitz et al., } \\
\text { USA [67] }\end{array}$} & Network of healthcare & Chiropractic care only & 52 & 49 & & 340 \\
\hline & & $\begin{array}{l}\text { Chiropractic care \& physical modalities } \\
\text { (Heat/cold, USS) }\end{array}$ & 53 & 58 & & 340 \\
\hline \multirow[t]{2}{*}{$\begin{array}{l}\text { Jellema et al., } \\
\text { Netherlands [68] }\end{array}$} & General practice & $\begin{array}{l}\text { Minimal intervention strategy (Assessing } \\
\text { psychosocial risks, providing information } \\
\text { on back pain and treatments \& advice } \\
\text { on self care) }\end{array}$ & 43 & 48 & 2 & 143 \\
\hline & & Usual GP care & 42 & 47 & 2 & 171 \\
\hline \multirow[t]{2}{*}{$\begin{array}{l}\text { Kaapa H., } \\
\text { Finland [69] }\end{array}$} & Occupational healthcare & $\begin{array}{l}\text { Multidisciplinary rehabilitation: guided, } \\
\text { group programme. : CBT, relaxation, } \\
\text { back school education \& physical therapy }\end{array}$ & 46 & 100 & 72 & 59 \\
\hline & & Individual physiotherapy & 47 & 100 & 63 & 61 \\
\hline \multirow[t]{2}{*}{$\begin{array}{l}\text { Kankaanpaa, Finland } \\
\text { [70] }\end{array}$} & Occupational healthcare & $\begin{array}{l}\text { Active rehabilitation: guided exercises } \\
\text { in a dept + behavioural support }\end{array}$ & 40 & 34 & & 30 \\
\hline & & $\begin{array}{l}\text { Passive treatment: which they considered } \\
\text { as minor to the active arm, e.g. massage } \\
\text { and thermal treatment }\end{array}$ & 39 & 33 & & 24 \\
\hline \multirow{2}{*}{$\begin{array}{l}\text { Kapitza et al., } \\
\text { Germany [71] }\end{array}$} & General population & Contingent biofeedback & 53 & 67 & 655 & 21 \\
\hline & & Non-contingent biofeedback (placebo) & 54 & 62 & 800 & 21 \\
\hline \multirow[t]{3}{*}{$\begin{array}{l}\text { Karjalainen et al., } 2003 \\
\& 2004 \text {, Finlands } \\
{[72,73]}\end{array}$} & General practice & $\begin{array}{l}\text { Mini-intervention (Specific back exercises, } \\
\text { reduce patient concerns \& encourage } \\
\text { physical activity) }\end{array}$ & 44 & 59 & & 56 \\
\hline & & Mini-intervention \& worksite visit & 44 & 57 & & 51 \\
\hline & & Usual GP care & 43 & 60 & & 57 \\
\hline \multirow{2}{*}{$\begin{array}{l}\text { Kennedy et al., } \\
\text { UK [74] }\end{array}$} & Primary care & Acupuncture + back book & 47 & 46 & & 24 \\
\hline & & Sham acupuncture + back book & 45 & 58 & & 24 \\
\hline
\end{tabular}


Table 2 Characteristics of included RCTs (n 70) (Continued)

\begin{tabular}{|c|c|c|c|c|c|c|}
\hline \multirow{2}{*}{$\begin{array}{l}\text { Kerr et al., } \\
\text { UK [75] }\end{array}$} & General practice & Acupuncture & 43 & 50 & 86 & 30 \\
\hline & & Placebo TENS (non-functioning) & 43 & 65 & 73 & 30 \\
\hline \multirow{3}{*}{$\begin{array}{l}\text { Kovacs et al., } \\
\text { Spain [76] }\end{array}$} & Nursing home consulters & Back book education & 80 & 66 & & 233 \\
\hline & & Back guide education & 81 & 63 & & 199 \\
\hline & & Pamphlet with cardiovascular health advice & 80 & 64 & & 241 \\
\hline \multirow[t]{3}{*}{$\begin{array}{l}\text { Kuukkanen et al., } \\
\text { Finland [77] }\end{array}$} & Occupational healthcare & $\begin{array}{l}\text { Intensive training: intensive progressive } \\
\text { exercises guided at the gym + home exercises }\end{array}$ & & 62 & & 29 \\
\hline & & $\begin{array}{l}\text { Home exercise only: same as intensive, } \\
\text { but unguided }\end{array}$ & & 48 & & 29 \\
\hline & & Control: usual activities, no trial exercises & & 54 & & 28 \\
\hline \multirow[t]{2}{*}{$\begin{array}{l}\text { Leclaire et al., } \\
\text { Canada [78] }\end{array}$} & Private physiatrist clinic & $\begin{array}{l}\text { Standard care (rest, analgesics, physio) \& } \\
\text { Swedish back school }\end{array}$ & 32 & 43 & & 82 \\
\hline & & Standard care (rest, analgesics, physio) & 32 & 41 & & 86 \\
\hline \multirow[t]{2}{*}{$\begin{array}{l}\text { Lindstrom et al., } \\
\text { Sweden [79] }\end{array}$} & Occupational healthcare & $\begin{array}{l}\text { Swedish back school \& workplace visit + } \\
\text { graded exercise (CBT approach) }\end{array}$ & & 24 & & 51 \\
\hline & & $\begin{array}{l}\text { Usual care: rest\& analgesics \& } \\
\text { physical treatment }\end{array}$ & & 38 & & 52 \\
\hline \multirow[t]{3}{*}{$\begin{array}{l}\text { Linton et al., } \\
\text { Sweden [80] }\end{array}$} & $\begin{array}{l}\text { General practice + general } \\
\text { population }\end{array}$ & Back pain pamphlet & 45 & 71 & & 70 \\
\hline & & Comprehensive information package & 44 & 74 & & 66 \\
\hline & & CBT intervention & 44 & 70 & & 107 \\
\hline \multirow{2}{*}{$\begin{array}{l}\text { Luijsterburg et al., } \\
\text { Netherlands [81] }\end{array}$} & Primary care & Physical therpay + GP care & 42 & 57 & & 67 \\
\hline & & Usual GP care & 43 & 40 & & 68 \\
\hline \multirow{2}{*}{$\begin{array}{l}\text { Machado et al., } \\
\text { Brazil [82] }\end{array}$} & Physiotherapy & Psychotherapy & 45 & 81 & 356 & 16 \\
\hline & & Exercise & 42 & 59 & 206 & 17 \\
\hline \multirow[t]{3}{*}{$\begin{array}{l}\text { Mannion et al., } 1999 \text { \& } \\
\text { 2001, Finland }[83,84]\end{array}$} & General population & $\begin{array}{l}\text { Modern active individual physiotherapy: } \\
\text { strengthening, coordination and aerobics } \\
\text { exercises, instructions on ergonomic } \\
\text { principles + home exercises }\end{array}$ & 46 & 61 & 520 & 46 \\
\hline & & $\begin{array}{l}\text { Muscle reconditioning on training devices } \\
\text { (small groups) }\end{array}$ & 45 & 54 & 504 & 47 \\
\hline & & Low impact aerobic/stretching (groups) & 44 & 55 & 676 & 44 \\
\hline \multirow{2}{*}{$\begin{array}{l}\text { Maul et al., } \\
\text { Switzerland [85] }\end{array}$} & Occupational healthcare & Back school \& exercise & 38 & & & 97 \\
\hline & & Back school & 39 & & & 86 \\
\hline \multirow{2}{*}{$\begin{array}{l}\text { Mehling et al., } \\
\text { USA [86] }\end{array}$} & General practice & Breath therapy & 50 & 70 & 51 & 16 \\
\hline & & $\begin{array}{l}\text { Physical therapy: soft tissue mobilisation, } \\
\text { joint mobilisation and exercises }\end{array}$ & 49 & 58 & 57 & 12 \\
\hline \multirow[t]{2}{*}{$\begin{array}{l}\text { Moseley L, } \\
\text { Australia [87] }\end{array}$} & $\begin{array}{l}\text { Physiotherapy }+ \text { General } \\
\text { practice }\end{array}$ & Physiotherapy & 43 & 64 & & 29 \\
\hline & & Usual GP care & 38 & 54 & & 28 \\
\hline \multirow{2}{*}{$\begin{array}{l}\text { Niemisto et al., } 2003 \text { \& } \\
\text { 2005, Finland }[88,89]\end{array}$} & General population & Manipulation, exercise \& physician consultation & 37 & 55 & 312 & 102 \\
\hline & & Physician consultation only & 37 & 53 & 312 & 102 \\
\hline \multirow[t]{2}{*}{$\begin{array}{l}\text { Nordeman et al., } \\
\text { Sweden [90] }\end{array}$} & $\begin{array}{l}\text { General practice + physical } \\
\text { therapy dept }\end{array}$ & $\begin{array}{l}\text { Early access to physio (Individualised, } \\
\text { exercise, advice, group education) }\end{array}$ & 39 & 63 & & 32 \\
\hline & & Waiting list control & 41 & 50 & & 28 \\
\hline
\end{tabular}


Table 2 Characteristics of included RCTs (n 70) (Continued)

\begin{tabular}{|c|c|c|c|c|c|c|}
\hline \multirow{3}{*}{$\begin{array}{l}\text { Paatelma et al., } \\
\text { Finland [91] }\end{array}$} & Occupational clinic & Orthopaedic manual therapy & 44 & 42 & & 45 \\
\hline & & McKenzie technique & 44 & 29 & & 52 \\
\hline & & Advice only & 44 & 35 & & 37 \\
\hline \multirow[t]{2}{*}{$\begin{array}{l}\text { Peloso et al., } \\
\text { USA [92] }\end{array}$} & Outpatients & $\begin{array}{l}\text { Tramadol \& Acetamenophen combination } \\
\text { tablets 375/325 } 2 \text { PRN }\end{array}$ & 58 & 64 & & 167 \\
\hline & & Placebo tablets 2 PRN & 58 & 61 & & 169 \\
\hline \multirow{3}{*}{$\begin{array}{l}\text { Rantonen et al., } \\
\text { Finland [93] }\end{array}$} & Occupational clinic & Physical therapy & 44 & 35 & 676 & 43 \\
\hline & & Exercise & 45 & 28 & 520 & 43 \\
\hline & & Back book education & 44 & 32 & 728 & 40 \\
\hline \multirow{2}{*}{$\begin{array}{l}\text { Rasmussen-Barr et al., } \\
\text { Sweden [94] }\end{array}$} & Physiotherapy & Graded exercises & 37 & 50 & 468 & 36 \\
\hline & & Advice and walking & 40 & 50 & 572 & 35 \\
\hline \multirow[t]{2}{*}{$\begin{array}{l}\text { Rasmussen-Barr et al., } \\
\text { Sweden [95] }\end{array}$} & Physiotherapy & $\begin{array}{l}\text { Stabilizing training (Individual) (Cognitive }+ \\
\text { stabilisation of spinal muscles) }\end{array}$ & 39 & 70 & & 24 \\
\hline & & $\begin{array}{l}\text { Manual treatment (Individual) (Other } \\
\text { muscles exercises, no manipulation) }\end{array}$ & 37 & 78 & & 23 \\
\hline \multirow{2}{*}{$\begin{array}{l}\text { Rittweger et al., } \\
\text { Germany [96] }\end{array}$} & General population & Isodynamic lumbar extension & 50 & 44 & 603 & 30 \\
\hline & & $\begin{array}{l}\text { Vibration exercise (On a machine with a } \\
\text { vibrating platform) }\end{array}$ & 54 & 52 & 754 & 30 \\
\hline \multirow{2}{*}{$\begin{array}{l}\text { Ritvanen et al., } \\
\text { Finland [97] }\end{array}$} & General population & Traditional chiropractic bone setting & 41 & 45 & & 33 \\
\hline & & Physical therapy & 42 & 43 & & 28 \\
\hline \multirow{2}{*}{$\begin{array}{l}\text { Rossignol et al., } \\
\text { Canada [98] }\end{array}$} & Workers compensation & Coordination of primary healthcare program & 37 & 33 & & 54 \\
\hline & board & Usual GP care & 38 & 23 & & 56 \\
\hline \multirow{2}{*}{$\begin{array}{l}\text { Sahin et al., } \\
\text { Turkey [99] }\end{array}$} & Physical therapy clinics & Back school & 47 & 75 & 30 & 75 \\
\hline & & Physical therapy & 51 & 78 & 32 & 75 \\
\hline \multirow[t]{2}{*}{$\begin{array}{l}\text { Soukup et al., } \\
\text { Norway [100] }\end{array}$} & $\begin{array}{l}\text { General practice + general } \\
\text { population + referrals }\end{array}$ & Mensediesk exercise group intervention & 40 & 53 & 676 & 34 \\
\hline & & Waiting list group & 40 & 49 & 578 & 35 \\
\hline \multirow{2}{*}{$\begin{array}{l}\text { Staal et al., \& Hlobil } \\
\text { et al., Netherlands } \\
{[101,102]}\end{array}$} & Occupational healthcare & Graded activity (Physiotherapy + OT) & 39 & 5 & 9 & 67 \\
\hline & & Usual OT care & 37 & 8 & 8 & 67 \\
\hline \multirow{3}{*}{$\begin{array}{l}\text { Torstensen et al., } \\
\text { Norway [103] }\end{array}$} & Social security offices & Medical exercise therapy (MET) & 42 & 52 & & 71 \\
\hline & & Conventional physiotherapy (CP) & 43 & 48 & & 67 \\
\hline & & Self exercise & 40 & 51 & & 70 \\
\hline \multirow{3}{*}{$\begin{array}{l}\text { Tsui et al., } \\
\text { Hong Kong [104] }\end{array}$} & Physiotherapy & Electro-acupuncture \& back exercise & 40 & 76 & 39 & 14 \\
\hline & & Electrical heat acupuncture + back exercise & 39 & 71 & 54 & 14 \\
\hline & & Back exercise only & 41 & 62 & 50 & 14 \\
\hline \multirow[t]{4}{*}{$\begin{array}{l}\text { Turner et al., } \\
\text { USA [105] }\end{array}$} & $\begin{array}{l}\text { General practice + } \\
\text { physicians + general } \\
\text { population }\end{array}$ & Relaxation training (group) & & & & 24 \\
\hline & & Cognitive therapy (group) & & & & 23 \\
\hline & & $\begin{array}{l}\text { Cognitive therapy \& Relaxation } \\
\text { training (group) }\end{array}$ & & & & 25 \\
\hline & & Waiting list control & & & & 30 \\
\hline
\end{tabular}


Table 2 Characteristics of included RCTs (n 70) (Continued)

\begin{tabular}{|c|c|c|c|c|c|c|}
\hline \multirow{3}{*}{$\begin{array}{l}\text { Unsgaard-Tondel et al., } \\
\text { Norway [106] }\end{array}$} & Primary care & Low load exercise & 41 & 81 & 312 & 36 \\
\hline & & High load sling exercise & 43 & 64 & 468 & 36 \\
\hline & & General exercise & 36 & 65 & 312 & 37 \\
\hline \multirow{2}{*}{$\begin{array}{l}\text { van der Roer et al., } \\
\text { Netherlands [107] }\end{array}$} & Physiotherapy & Intensive protocol training & 42 & 55 & 54 & 60 \\
\hline & & Guidelines based physiotherapy & 42 & 48 & 47 & 54 \\
\hline \multirow[t]{2}{*}{$\begin{array}{l}\text { Wand et al., } \\
\text { UK [108] }\end{array}$} & $\begin{array}{l}\text { General practice + A/E } \\
\text { patients }\end{array}$ & Assess \& Advice \& Physiotherapy & 34 & 44 & & 43 \\
\hline & & Assess \& Advice \& wait & 35 & 55 & & 51 \\
\hline \multirow[t]{2}{*}{$\begin{array}{l}\text { Werners et al., } \\
\text { Germany [109] }\end{array}$} & General practice & $\begin{array}{l}\text { Interferential therapy: electrotherapy, } \\
\text { to stimulate muscles fibres }\end{array}$ & 38 & 43 & & 68 \\
\hline & & Motorised lumbar traction \& massage & 39 & 49 & & 72 \\
\hline \multirow{4}{*}{$\begin{array}{l}\text { Yelland et al., } \\
\text { Australia [110] }\end{array}$} & General practice & Glucose lignocaine injection & 52 & 59 & 770 & 28 \\
\hline & & $\begin{array}{l}\text { Exercise (Alternating: flexion and extension } \\
\text { of spine and hips) }\end{array}$ & 49 & 55 & 718 & 26 \\
\hline & & Saline injection & 50 & 56 & 759 & 27 \\
\hline & & Normal activity & 51 & 58 & 733 & 29 \\
\hline
\end{tabular}

and physiotherapy departments (19 RCTs). Eight trials were conducted among the general population and 10 in mixed settings. 13 RCTs (34 treatment arms) were classified by one of the authors (MA) as efficacy trials and the remaining 57 (131 treatment arms) as pragmatic trials. Eight RCTs included 'usual care' arms. The19 observational studies included consulters in general practice (11 studies) and other allied primary care services such as chiropractic clinics and physiotherapy departments, as well as cohorts sampled from the general population in two studies. All participants were described in the papers as receiving 'usual' or 'standard care'.

\section{The course of pain intensity scores over time}

Pooled mean pain intensity scores at baseline and follow up for RCTs and observational studies are presented in Figure 3 and Table 4. They show a similar pattern of symptom change over time in both groups. This is represented by a substantial rapid early improvement of mean pain intensity within the first 13 weeks of followup followed by a smaller further improvement over the follow-up period to 52 weeks.

Regarding the size of symptom change over time, pooled SMCs (Table 5) confirm the substantial improvement in pain symptoms in both groups. These range from 0.9 to 1.2 for RCTs and from 1.0 to 1.2 for observational studies.

There was a large between-study variation in the sizes of pain improvement from baseline within both observational studies and RCT treatment arms demonstrated by the high $I^{2}$ values (99\%).
Meta-regression analysis showed no statistically significant difference in the change in pain intensity (SMC) between all RCTs and observational studies at any follow up point. There was also no statistically significant difference in the change in pain intensity when considering the two types of RCTs (pragmatic and efficacy) separately compared with observational studies. Comparing cohort studies and usual care arms of RCTs also did not show any difference in the pattern or course of LBP between these groups.

\section{Discussion}

This study directly compared the course of non-specific low back pain symptoms in observational studies with

Table 3 Comparison of population characteristics of included RCTs and observational cohort studies

\begin{tabular}{|c|c|c|c|}
\hline & & Cohort studies & $\mathrm{RCTs}^{\mathrm{a}}$ \\
\hline Publication year & & 1994-2012 & $1993-2012$ \\
\hline \multirow{2}{*}{$\begin{array}{l}\text { Sample size, } \\
\text { Median (range) }\end{array}$} & & \multirow[t]{2}{*}{$368(60,2872)$} & $128(28,681)^{\mathrm{b}}$ \\
\hline & & & $67(12,340)^{c}$ \\
\hline Age, mean ${ }^{d}(S D)$ & & $43(4.1)$ & $44(7.9)$ \\
\hline $\begin{array}{l}\text { Female, mean } \\
\text { percentage (SD) }\end{array}$ & & $52(4.8)$ & $53(16.9)$ \\
\hline \multirow[t]{4}{*}{ Type of pain, n (\%) } & Acute & $5(26)$ & $34(20)$ \\
\hline & Chronic & $2(11)$ & $94(57)$ \\
\hline & Mixed & $12(63)$ & 31 (19) \\
\hline & Unclear & 0 & $6(4)$ \\
\hline $\begin{array}{l}\text { Baseline pain intensity, } \\
\text { mean }^{d} \text { (SD) }\end{array}$ & & $49.6(12.7)$ & $49.9(12.9)$ \\
\hline
\end{tabular}

${ }^{\mathrm{a}} \mathrm{RCTs}$ that provided data on pain intensity outcome. ${ }^{\mathrm{b}}$ Sample size of RCT. cSample size of arm. ${ }^{\mathrm{d}}$ Mean of all cohort/RCT means. 


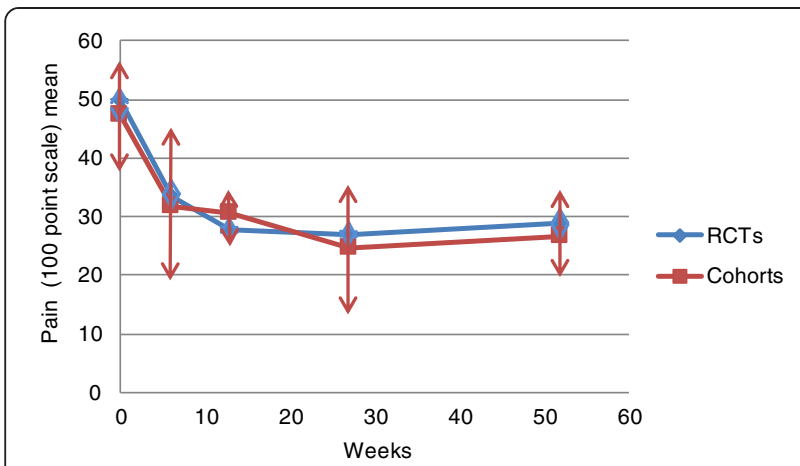

Figure 3 Pooled mean pain intensity scores (95\% confidence interval) for the included RCTs and observational cohort studies from baseline to 52 week follow up.

RCTs on primary care treatments for back pain. The results showed no significant difference in the size of symptom improvement and the pattern of this improvement over time.

Investigating whether any difference is concentrated between observational studies and efficacy RCTs failed to show any difference in the size of symptom improvement. This was to test the assumption that compared with pragmatic RCTs, efficacy RCTs are characterised by higher level of attention and adherence to treatment protocol as well as stricter criteria for patient selection and inclusion [111,112]. Guidelines and tools are available to describe clinical trials as efficacy or pragmatic. The purpose of some of these tools is to inform trial design [111] while others are for the purpose of systematic reviews [112]. RCTs, however, are very rarely purely pragmatic or efficacy trials and could often be described along a continuum between these two ends and most include features of both with possible dominance of either. To satisfy the specific aims of our study related to the care and attention received in studies, the approach adopted was to describe trials that included placebo, sham or no treatment arms as efficacy trials.
A separate comparison between observational studies and the 'usual treatment' arms of RCTs was assumed to provide a comparison of groups receiving similar types of treatments. This comparison also failed to show any difference in the pattern or size of the clinical course of symptoms in these groups. This echoes what we have previously demonstrated of the absence of a significant difference in the pattern or size of symptom improvement in RCTs comparing usual care with active treatment arms [4].

One of the findings in this study was the large heterogeneity among cohort studies and RCT arms. Conducting meta-analysis in the presence of a large heterogeneity is potentially problematic. Using random effects model would have ameliorated this problem to an extent, but not completely. For this reason, the outcome of the meta-analysis will need to be interpreted within the specific context and aim of this study, namely to study the general trend of the clinical course of symptoms. The heterogeneity could be explained by a number of potential methodological as well as clinical characteristics. Formally studying such potential sources of heterogeneity is important and is beyond the aims of this study.

Meta-analyses comparing RCTs and observational studies have been conducted with varying aims including comparing treatment effects [111], adverse effects of treatments [112,113] and prognostic factors [114]. However, although the clinical course of low back pain has been studied in observational studies $[10,11]$, we are not aware of a direct comparison with the clinical course of symptoms in RCTs. Furlan et al. [12] compared matching pairs of RCTs and non-randomised studies and included cohort studies but only those that had comparison groups. More significantly, the main aim of Furlan et al's work was to compare RCTs with non-randomised studies regarding their methodological quality rather than to study the clinical course of symptoms.

A number of factors have been suggested to influence the course of symptoms in clinical trials, related to the

Table 4 Pooled mean pain intensity scores $(95 \% \mathrm{Cl})$ for included $\mathrm{RCTs}$ and observational cohort studies using random effects meta-analysis

\begin{tabular}{|c|c|c|c|c|c|}
\hline & Baseline & 6 weeks & 13 weeks & 27 weeks & 52 weeks \\
\hline \multicolumn{6}{|l|}{ RCTs } \\
\hline Pain & $48.1(45.8,50.5)$ & $34.1(31.0,37.2)$ & $27.8(25.1,30.6)$ & $26.4(24.3,28.6)$ & $28.9(25.7,32.0)$ \\
\hline Arms, $n$ & 165 & 58 & 94 & 97 & 78 \\
\hline Sample size* & 10655 & 3577 & 6109 & 6640 & 4499 \\
\hline \multicolumn{6}{|l|}{ Cohorts } \\
\hline Pain & $47.3(38.6,56.0)$ & $31.7(18.5,44.8)$ & $30.7(25.6,35.8)$ & $24.7(12.9,36.4)$ & $26.7(19.8,33.6)$ \\
\hline $\mathrm{n}$ & 19 & 6 & 10 & 10 & 12 \\
\hline Sample size* & 13096 & 6122 & 6848 & 5496 & 6284 \\
\hline
\end{tabular}

*The total number of participants included in trials or cohort studies providing data for the analysis. 
Table 5 Pooled estimates of SMCs (95\% confidence interval) for pain intensity for included RCTs and observational cohort studies

\begin{tabular}{|c|c|c|c|c|c|c|c|c|c|c|c|c|}
\hline & & & & & & led $S$ & Cs $(95$ & $\mathrm{Cl})$ & & & & \\
\hline & & 6 weeks & & & 13 weeks & & & 27 weeks & & & 52 weeks & \\
\hline & $\overline{n^{\dagger}}$ & & $I^{2}$ & $\overline{n^{\dagger}}$ & & $I^{2}$ & $\overline{n^{\dagger}}$ & & $I^{2}$ & $\overline{n^{\dagger}}$ & & $1^{2}$ \\
\hline Cohorts & 6 & $1.2(0.7,1.7)$ & 99 & 9 & $1.0(0.8,1.3)$ & 99 & 9 & $1.2(0.8,1.7)$ & 99 & 11 & $1.1(0.8,1.6)$ & 99 \\
\hline RCTs & 60 & $1.0(0.9,1.0)$ & 99 & 94 & $1.2(1.1,1.3)$ & 100 & 101 & $1.1(1.0,1.2)$ & 100 & 78 & $0.9(0.8,1.0)$ & 99 \\
\hline$p$-value* & & 0.651 & & & 0.735 & & & 0.878 & & & 0.721 & \\
\hline Efficacy RCTs & 15 & $1.0(0.9,1.1)$ & 99 & 13 & $1.2(1.0,1.4)$ & 100 & 16 & $0.9(0.7,1.2)$ & 100 & 14 & $0.7(0.5,0.8)$ & 100 \\
\hline$p$-value $e^{* *}$ & & 0.663 & & & 0.549 & & & 0.574 & & & 0.104 & \\
\hline Pragmatic RCTs & 43 & $1.0(0.9,1.1)$ & 99 & 81 & $1.2(1.1,1.4)$ & 100 & 81 & $1.2(1.0,1.3)$ & 100 & 64 & $0.9(0.8,1.1)$ & 100 \\
\hline$p$-value ${ }^{* * *}$ & & 0.628 & & & 0.466 & & & 0.899 & & & 0.642 & \\
\hline Usual Care RCT arms & & & & 8 & $1.2(1.0,1.3)$ & 99 & 7 & $1.3(1.7,1.4)$ & 99 & 7 & $1.0(0.8,1.2)$ & 99 \\
\hline
\end{tabular}

${ }^{\dagger}$ Number of cohort studies and RCTs treatment arms. * Meta-regression comparison between cohort studies and RCTs. efficacy RCTs, ${ }^{* *}$ Meta-regression comparison between cohort studies and efficacy RCTs. ${ }^{* * *}$ Meta-regression comparison between cohort studies and pragmatic RCTs.

participants (e.g. cultural background, health literacy) [115-117], the practitioner/researcher (e.g. communication skills and experience with the use of the treatment) $[115,118]$ and the characteristics of the treatment (e.g. invasiveness, physical contact and psychological component) [119]. Another factor is suggested to relate to the actual enrolment in a trial. This is assumed to be related to the factual and perceived extensive care and attention provided in the trial - the 'Hawthorne effect', the 'care effect' or the unique strict adherence to the treatment protocol 'protocol effect'. Such effects are assumed to contribute to extra improvement among participants in clinical trials compared with other studies or usual clinical practice [5].

The clinical course of back pain in observational studies might simply represent an extension of our earlier findings in RCTs [4]. This represents an average 'general response to health care' which dominates any individual responses to treatments. This general response overwhelms any additional effect of being in a trial, observational study or in fact seeking usual routine care. It is true that specific treatments are provided in RCTs as opposed to observational studies where no particular treatments are specified. In fact none of the observational studies included in our review included a specific treatment. However, conservative treatments for non-specific low back pain investigated in RCTs are not new but already available in clinical practice $[1,3]$. This might mean that expectations of novel and big effects among those participating in RCTs of back pain are not generally high.

Alternatively, differences may exist between RCTs and observational studies in the care and attention provided. But the effect on the clinical course of symptoms lies in outcomes other than those captured by pain intensity. Outcomes that may specifically represent components of a 'trial effect', and their measurement was beyond the scope of this paper.
Participants of observational studies are arguably similar to patients presenting in usual clinical practice. This means that our findings suggest that RCTs participants are not different from the average patients with regard to the clinical course of LBP. This challenges the assumption that participants in clinical trials are somehow different from the average patients. Or that their symptoms run a course that is to an extent influenced by mere participation in the trial. In other words, or findings would support the generalizability of the trials' findings to patients in usual clinical practice. The findings also throws in doubt the assumption related to the effect of mere participation in a trial, although our study did not specifically aims to study this effect.

\section{Limitations}

A large number of observational studies and RCTs on a wide range of treatments for non-specific low back pain were included to study the overall size of change in pain symptoms over time. The study, however, has a number of limitations.

For literature search, we adopted the same strategy that was adopted in a previous study conducted and published by the same group to examine the course of LBP in RCTs [4]. This was an updated access to the CENTRAL database. Although this might have limited the number of RCTs included in the study, it is unlikely that this represented a very large number that would have impacted the study outcome. Adopting the same strategy also provides the opportunity for a continuity of comparison between the two studies.

Also, as the aim of the study was to investigate the overall clinical course of LBP rather than to estimate the effectiveness of a particular treatment, an exhaustive inclusion of all trials on back pain treatments was not required. The aim was to have a large and representative pool of clinical trials that would vary sufficiently with 
respect to the types of treatments to achieve the objectives in this review and the CENTRAL database satisfied this aim. As a similar data base does not exist for observational cohort, a different search strategy was conducted for this group of studies.

The numbers of included RCTs and observational studies were not comparable. This might raise the concern that the outcome of the comparison is inaccurate. Although this is an arguably valid concern, the comparison with smaller subgroups of RCTs (efficacy RCTs and usual care arms) provided a more comparable numbers. The outcome of these comparisons confirmed the outcome of comparing the total groups of RCTs and cohort studies, which should help alleviate the related concerns.

The focus in our study was on pain intensity outcome using a Numerical Rating Scale (NRS) or Visual Analogue Scale (VAS). This was because of the lack of data on other outcome measures such as functional disability outcomes that would allow for a satisfactory comparison. The forced focus on one outcome measure in meta-analysis is common in systematic reviews of observational studies because of the lack of data on other outcome measures [11]. Excluding studies that did not provide data relevant to the analysis used in this study might have influenced our results. However, we have no evidence to suggest that this has led to systematic exclusion of studies with either large or small improvement in symptoms. We found in a previous review that the overall course of symptoms using functional disability outcomes (Roland Morris disability questionnaire, RMDQ and Oswestry Disability Inventory ODI) was similar to that when using pain intensity outcome [4].

\section{Conclusion}

The course of back pain symptoms in observational studies follows a pattern that is similar to that in RCTs, notably in the size of the average improvement in pain intensity over time. This suggests that, in both types of studies, a general improvement in back pain symptoms and comparable responses to nonspecific effects related to seeking and receiving care occur regardless of the study design.

\section{Additional file}

Additional file 1: Literature search strategy for observationa cohort studies.

\section{Competing interest}

The author(s) declare that they have no competing interests.

\section{Authors' contribution}

This study was part of a larger research project for the PhD conducted by MA, supervised by DvdW and KPJ. The PhD project was funded through an Arthritis Research UK Primary Care Fellowship, number 17890. All authors contributed equally to writing the article and all authors read and approved the final manuscript.

Received: 17 August 2013 Accepted: 25 February 2014

Published: 7 March 2014

\section{References}

1. van Tulder MW, Koes BW, Malmivaara A: Outcome of non-invasive treatment modalities on back pain: an evidence-based review. Eur Spine J 2006, 15(Suppl 1):S64-S81.

2. Keller A, Hayden J, Bombardier C, Van Tulder M: Effect sizes of non-surgical treatments of non-specific low back pain. Eur Spine J 2007, 16:1776-1788.

3. Machado LAC, Kamper SJ, Herbert RD, Maher CG, MacAuley JH: Analgesic effects of treatments for non-specific low back pain: a meta-analysis of placebo controlled randomized trials. Rheumatology 2009, 48:520-527.

4. Artus M, van der Windt DA, Jordan KP, Hay EM: Low back pain symptoms show a similar pattern of improvement following a wide range of primary care treatments: a systematic review of randomized clinical trials. Rheumatology 2012, 49:2346-2356.

5. Pengel $L$, Herbert $R$, Maher $C$, Refshauge K: Acute low back pain: systematic review of its prognosis. BMJ 2003, 327:323-328.

6. Hestbaek L, Leboeuf-Yde C, Manniche C: Low back pain: what is the long-term course? A review of studies of general patient populations. Eur Spine J 2003, 12:149-165.

7. Furlan AD, Tomlinson G, Jadad AR, Bombardier C: Methodological quality and homogeneity influenced agreement between randomized trials and nonrandomized studies of the same intervention for back pain. J Clin Epidem 2008, 61:209-231.

8. Braunholtz DA, Edwards SJL, Lilford RJ: Are randomized clinical trials good for us (in the short term)? Evidence for a "trial effect. J Clin Epi 2001, $54: 217-224$

9. Vist GE, Hagen KB, Devereaux PJ, Bryant D, Kristoffersen DT, Oxman AD: Systematic review to determine whether participation in a trial influences outcome. BMJ 2005, 330:21.

10. Benedetti F, Pollo A, Lopiano L, Lanotte M, Vighetti S, Rainero I: Conscious Expectation and unconscious conditioning in analgesic, motor, and hormonal placebo/nocebo responses. J Neuroscience 2003, 23:4315-4323.

11. Benedetti F, Mayberg HS, Wager TD, Stohler CS, Zubieta JK5: Neurobiological mechanisms of the placebo effect. J Neurosci 2005, 25:10390-10402

12. Kaptchuk TJ, Kelley JM, Conboy LA, Davis RB, Kerr CE, Jacobson EE, Kirsch I, Schyner RN, Nam BH, Nguyen LT, Park M, Rivers AL, McManus C, Kokkotou E, Drossman DA, Goldman P, Lembo AJ: Components of placebo effect: randomised controlled trial in patients with irritable bowel syndrome. BMJ 2008, 336:999-1003.

13. Morris SB: Distribution of the standardized mean change effect size for meta-analysis on repeated measures. B J Math Stat Psych 2000, 53:17-29.

14. Cohen J: Statistical Power Analysis for the Behavioural Sciences. 2nd edition. Hillsdale, New Jersey: Lawrence Erlbaum Associates, Inc:; 1998.

15. Hozo SP, Djulbegovic B, Hozo I: Estimating the mean and variance from the median, range, and the size of a sample. BMC Med Res Methodol 2005, 5:c13.

16. Higgins JP, Thompson SG, Deeks JJ, Altman DG: Measuring inconsistency in meta-analyses. BMJ 2003, 327:557-560.

17. Bakker EWP, Verhagen AP, Lucas C, Koning HJCM, Koes BE: Spinal mechanical load: a predictor of persistent low back pain? A prospective cohort study. Eur Spine J 2006, 16:933-941. Cohort studies reference List (for web publishing only).

18. Bekkering GE, Hendriks HJM, van Tulder MW, Knol DL, Simmonds MJ, Oostendorp RAB, Bouter LM: Prognostic factors for low back pain in patients referred for physiotherapy: Comparing outcomes and varying modeling techniques. Spine 2005, 30:1881-1886. Cohort studies reference List (for web publishing only).

19. Carey TS, Garrett J, Jackman A, McLaughlin C, Fryer J, Smucker DR: The outcomes and costs of care for acute low back pain among patients seen by primary care practitioners, chiropractors, and orthopedic surgeons. NEJM 1995, 333:913-917. Cohort studies reference List (for web publishing only).

20. Chenot JF, Becker A, Leonhardt C, Keller S, Donner-Banzhoff N, Hildebrandt J, Basler HD, Baum E, Kochen MM, Pfingsten M: Sex differences in presentation, 
course, and management of low back pain in primary care. Clin J Pain 2008, 24:578-584. Cohort studies reference List (for web publishing only).

21. Coste J, Delecoeuillerie G, Cohen A, Le JM, Paolaggi JB: Clinical course and prognostic factors in acute low back pain: an inception cohort study in primary care practice. BMJ 1994, 308:577-580. Cohort studies reference List (for web publishing only).

22. Demmelmaier I, Åsenlöf P, Lindberg P, Denison E: Biopsychosocial predictors of pain, disability, health care consumption, and sick leave in first-episode and long-term back pain: a longitudinal study in the general population. Int J Behav Med 2010, 17:79-89. Cohort studies reference List (for web publishing only).

23. Dunn KM, Jordan KP, Croft PR: Contributions of prognostic factors for poor outcome in primary care low back pain patients. Euro J Pain 2011, 15:313-319. Cohort studies reference List (for web publishing only).

24. Grotle M, Brox Jl, Veierod MB, Glomsrod B, Lonn JH, Vollestad NK: Clinical course and prognostic factors in acute low back pain: patients consulting primary care for the first time. Spine 2005, 30:976-982. Cohort studies reference List (for web publishing only).

25. Haas M, Goldberg B, Aickin M, Ganger B, Attwood M: A practice-based study of patients with acute and chronic low back pain attending primary care and chiropractic physicians: two-week to 48-month follow-up. J Manip Physiol Ther 2004, 27:160-169. Cohort studies reference List (for web publishing only).

26. Kovacs FM, Fernandez C, Cordero A, Muriel A, Gonzalez-Lujan L, Gil MT: Non-specific low back pain in primary care in the Spanish National Health Service: a prospective study on clinical outcomes and determinants of management. BMC Health Serv Res 2006, 6:C57. Cohort studies reference List (for web publishing only).

27. McGuirk B, King W, Govind J, Lowry J, Bogduk N: Safety, efficacy, and cost effectiveness of evidence-based guidelines for the management of acute low back pain in primary care. Spine 2001, 26:2615-2622. Cohort studies reference List (for web publishing only).

28. Miller P, Kendrick D, Bentley E, Fielding K: Cost-effectiveness of lumbar spine radiography in primary care patients with low back pain. Spine 2002, 27:2291-2297. Cohort studies reference List (for web publishing only).

29. Nyiendo J, Haas M, Goldberg B, Sexton G: Pain, disability, and satisfaction outcomes and predictors of outcomes: a practice-based study of chronic low back pain patients attending primary care and chiropractic physicians. J Manip Physiol Ther 2001, 24:433-439. Cohort studies reference List (for web publishing only).

30. Perreault K, Dionne CE: Does patient-physiotherapist agreement influence the outcome of low back pain? A prospective cohort study. BMC Musculoskelet Disord 2006, 7:c76. Cohort studies reference List (for web publishing only)

31. Seferlis T, Nemeth G, Carlsson AM, Gillstrom P: Conservative treatment in patients sick-listed for acute low-back pain: a prospective randomised study with 12 months' follow-up. Eur Spine J 1998, 7:461-470. Cohort studies reference List (for web publishing only).

32. Sharma R, Haas M, Stano M, Spegman A, Gehnng R: Determinants of costs and pain improvement for medical and chiropractic care of low back pain. J Manip Physiol Ther 2009, 32:252-261. Cohort studies reference List (for web publishing only).

33. Tamcan O, Mannion AF, Eisenring C, Horisberger B, Elfering A, Muller U: The course of chronic and recurrent low back pain in the general population. Pain 2010, 150:451-457. Cohort studies reference List (for web publishing only).

34. van den Hoogen HJM, Koes BW, van Eijk JM, Bouter LM, Devillé W: On the course of low back pain in general practice: a one year follow up study. Ann Rheum Dis 1998, 57:13-19. Cohort studies reference List (for web publishing only).

35. van Tulder MW, Koes BW, Metsemakers JF, Bouter LM: Chronic low back pain in primary care: a prospective study on the management and course. Fam Pract 1998, 15:126-132. Cohort studies reference List (for web publishing only).

36. Albaladejo C, Kovacs FM, Royuela A, del Pino R, Zamora J, Spanish Back Pain Research Network: The efficacy of a short education program and a short physiotherapy program for treating low back pain in primary care: a cluster randomized trial. Spine 2010, 35:483-496. RCTs reference List (for web publication only).

37. Arribas MJD, Sanchez MR, Herva PP, Chicharro JL, Carrere TA, Molina PO, Arbiza PA: Effectiveness of the physical therapy Godelive Denys-Struyf method for nonspecific low back pain primary care randomized control trial. Spine 2009, 34:1529-1538. RCTs reference List (for web publication only).

38. Bendix T, Bendix A, Labriola M, Haestrup C, Ebbehøj N: Functional restoration versus outpatient physical training in chronic low back pain: a randomized comparative study. Spine 2000, 25:2494-2500. RCTs reference List (for web publication only).

39. Bronfort G, Goldsmith CH, Nelson CF, Boline PD, Anderson AV: Trunk exercise combined with spinal manipulative or NSAID therapy for chronic low back pain: a randomized, observer-blinded clinical trial. J Manipulative Physiol Ther 1996, 19:570-582. RCTs reference List (for web publication only).

40. Bronfort G, Maiers MJ, Evans RL, Schulz CA, Bracha Y, Svendsen KH, Grimm RH Jr, Owens EF Jr, Garvey TA, Transfeldt EE: Supervised exercise, spinal manipulation, and home exercise for chronic low back pain: a randomized clinical trial. Spine J 2011, 11:585-598. RCTs reference List (for web publication only).

41. Browder DA, Childs JD, Cleland JA, Fritz JM: Effectiveness of an extension-oriented treatment approach in a subgroup of subjects with low back pain: a randomized clinical trial. Phys Ther 2007, 87:1608-1618. RCTs reference List (for web publication only).

42. Burton AK, Waddell G, Tillotson KM, Summerton N: Information and advice to patients with back pain can have a positive effect. A randomised controlled trial of a novel educational booklet in primary care. Spine 1999, 24:2484-2491. RCTs reference List (for web publication only).

43. Cambron JA, Gudavalli MR, Hedeker D, McGregor M, Jedlicka J, Keenum M, Ghanayem AJ, Patwardhan AG, Furner SE: One-year follow-up of a randomized clinical trial comparing flexion distraction with an exercise program for chronic low-back pain. J Alt Comp Med 2006, 12:659-668. RCTs reference List (for web publication only).

44. Cecchi F, Molino-Lova R, Chiti M, Pasquini G, Gnocchi AP, Conti AA, Macchi C: Spinal manipulation compared with back school and with individually delivered physiotherapy for the treatment of chronic low back pain: a randomized trial with one-year follow-up. Clin Rehab 2010, 24:26-36. RCTs reference List (for web publication only).

45. Chan CW, Mok NW, Yeung EW: Aerobic exercise training in addition to conventional physiotherapy for chronic low back pain: a randomized controlled trial. Arch Phys Med Rehab 2011, 92:1681-1685. RCTs reference List (for web publication only).

46. Chang ST, Chen LC, Chang CC, Chu HY, Hsieh MF, Tsai KC: Efficacy and safety of piroxicam beta-cyclodextrin sachets for treating chronic low back pain: a randomized, parallel, active-controlled trial. J Med Sci 2008, 28:111-119. RCTs reference List (for web publication only).

47. Chok B, Lee R, Latimer J, Tan SB: Endurance training of the trunk extensor muscles in people with subacute low back pain. Phys Ther 1999, 79:1032-1042. $\mathrm{RCTs}$ reference List (for web publication only).

48. Costa LO, Maher CG, Latimer J, Hodges PW, Herbert RD, Refshauge KM, McAuley JH, Jennings MD: Motor control exercise for chronic low back pain: a randomized placebo-controlled trial. Phys Ther 2009, 89:1275-1286. $\mathrm{RCT}$ s reference List (for web publication only).

49. Constant F, Collin JF, Guillemin F, Boulangé M: Effectiveness of spa therapy in chronic low back pain: a randomized clinical trial. J Rheumatol 1995, 22:1315-1320. RCTs reference List (for web publication only).

50. Critchley DJ, Ratcliffe J, Noonan S, Jones RH, Hurley MV: Effectiveness and costeffectiveness of three types of physiotherapy used to reduce chronic low back pain disability: a pragmatic randomized trial with economic evaluation. Spine 2007, 32:1474-1481. RCTs reference List (for web publication only).

51. Di Cesarea A, Giombinib A, Di Cesarec M, Ripanib M, Vulpiani MC, Saracenia VM: Comparison between the effects of trigger point mesotherapy versus acupuncture points mesotherapy in the treatment of chronic low back pain: A short term randomized controlled trial. Compl Ther in Med 2011, 19:19-26. RCTs reference List (for web publication only).

52. Djavid GE, Mehrdad R, Ghasemi M, Hasan ZH, Sotoodeh MA, Pouryaghoub G In chronic low back pain, low level laser therapy combined with exercise is more beneficial than exercise alone in the long term: a randomised trial. Australian J Physio 2007, 53:155-160. RCTs reference List (for web publication only).

53. Dufour N, Thamsborg G, Oefeldt A, Lundsgaard C, Stender S: Treatment of chronic low back pain: a randomized, clinical trial comparing group-based multidisciplinary biopsychosocial rehabilitation and intensive individual therapist-assisted back muscle strengthening exercises. Spine 2010, 35:469-476. RCTs reference List (for web publication only). 
54. Dundar U, Solak O, Yigit I, Evcik D, Kavuncu V: Clinical effectiveness of aquatic exercise to treat chronic low back pain: a randomized controlled trial. Spine 2009, 34:1436-1440. RCTs reference List (for web publication only).

55. Fritz JM, Lindsay W, Matheson JW, Brennan GP, Hunter SJ, Moffit SD, Swalberg A, Rodriquez B: Is there a subgroup of patients with low back pain likely to benefit from mechanical traction? Results of a randomized clinical trial and subgrouping analysis. Spine 2007, 32:E793-E800. RCTs reference List (for web publication only).

56. Frost H, Lamb SE, Doll HA, Carver PT, Stewart BS: Randomised controlled trial of physiotherapy compared with advice for low back pain. BMJ 2004, 329: C708. RCTs reference List (for web publication only).

57. Geisser ME, Wiggert EA, Haig AJ, Colwell MO: A randomized, controlled trial of manual therapy and specific adjuvant exercise for chronic low back pain. Clin J Pain 2005, 21:463-470. RCTs reference List (for web publication only).

58. George SZ, Fritz JM, Bialosky JE, Donald DA: The effect of a fear-avoidancebased physical therapy intervention for patients with acute low back pain: results of a randomized clinical trial. Spine 2003, 28:2551-2560. RCTs reference List (for web publication only).

59. Glazov G, Schattner P, Lopez D, Shandley K: Laser acupuncture for chronic non-specific low back pain: a controlled clinical trial. Acupuncture Med 2009, 27:94-100. RCTs reference List (for web publication only).

60. Glomsrød B, Lønn JH, Soukup MG, Bø K, Larsen S: "Active back school", prophylactic management for low back pain: three-year follow-up of a randomized, controlled trial. J Rehab Med 2001, 33:26-30. RCTs reference List (for web publication only).

61. Goldby LJ, Moore AP, Doust J, Trew ME: A randomized controlled trial investigating the efficiency of musculoskeletal physiotherapy on chronic low back disorder. Spine 2006, 31:1083-1093. RCTs reference List (for web publication only).

62. Hay EM, Mullis R, Lewis M, Vohora K, Main CJ, Watson P, Dziedzic KS, Sim J, Minns Lowe C, Croft PR: Comparison of physical treatments versus a brief pain-management programme for back pain in primary care: a randomised clinical trial in physiotherapy practice. Lancet 2005, 365:2024-2030. RCTs reference List (for web publication only).

63. Heymans MW, de-Vet HC, Bongers PM, Knol DL, Koes BW, van Mechelen W The effectiveness of high-intensity versus low-intensity back schools in an occupational setting: a pragmatic randomized controlled trial. Spine 2006, 31:1075-1082. RCTs reference List (for web publication only).

64. Hsieh CY, Adams AH, Tobis J, Hong CZ, Danielson C, Platt K, Hoehler F, Reinsch S, Rubel A: Effectiveness of four conservative treatments for subacute low back pain: a randomized clinical trial. Spine 2002, 27:1142-1148. RCTs reference List (for web publication only).

65. Hurley DA, McDonough SM, Dempster M, Moore AP, Baxter GD: A randomized clinical trial of manipulative therapy and interferential therapy for acute low back pain. Spine 2004, 29:2207-2216. RCTs reference List (for web publication only).

66. Hurwitz EL, Morgenstern H, Harber P, Kominski GF, Belin TR, Yu F, Adams $\mathrm{AH}$ : A randomized trial of medical care with and without physical therapy and chiropractic care with and without physical modalities for patients with low back pain: 6-month follow-up outcomes from the UCLA low back pain study. Spine 2002, 27:2193-2204. RCTs reference List (for web publication only).

67. Hurwitz EL, Morgenstern H, Harber P, Kominski GF, Belin TR, Yu F, Adams $\mathrm{AH}$ : The effectiveness of physical modalities among patients with low back pain randomized to chiropractic care: findings from the UCLA low back pain study. J Manip Physiol Ther 2002, 25:10-20. RCTs reference List (for web publication only).

68. Jellema P, van der Windt DA, van der Horst HE, Twisk JW, Stalman WA, Bouter LM: Should treatment of (sub)acute low back pain be aimed at psychosocial prognostic factors? Cluster randomised clinical trial in general practice. BMJ 2005, 331:84. RCTs reference List (for web publication only).

69. Kääpä EH, Frantsi K, Sarna S, Malmivaara A: Multidisciplinary group rehabilitation versus individual physiotherapy for chronic nonspecific low back pain: a randomized trial. Spine 2006, 31:371-376. RCTs reference List (for web publication only).

70. Kankaanpää M, Taimela S, Airaksinen $\mathrm{O}$, Hänninen $\mathrm{O}$ : The efficacy of active rehabilitation in chronic low back pain. Effect on pain intensity, self-experienced disability, and lumbar fatigability. Spine 1999, 24:1034-1042. RCTs reference List (for web publication only).
71. Kapitza KP, Passie T, Bernateck M, Karst M: First non-contingent respiratory biofeedback placebo versus contingent biofeedback in patients with chronic low back pain: a randomized, controlled, double-blind trial. Appl Psychophysiol Biofeedback 2010, 35:207-217. RCTs reference List (for web publication only).

72. Karjalainen $\mathrm{K}$, Malmivaara A, Pohjolainen $T$, Hurri H, Mutanen $\mathrm{P}$, Rissanen $\mathrm{P}$, Pahkajärvi H, Levon H, Karpoff H, Roine R: Mini-intervention for subacute low back pain: a randomized controlled trial. Spine 2003, 28:533-540. $\mathrm{RCT}$ s reference List (for web publication only).

73. Karjalainen $K$, Malmivaara A, Mutanen $P$, Roine $R$, Hurri H, Pohjolainen $T$ : Mini-intervention for subacute low back pain: two-year follow-up and modifiers of effectiveness. Spine 2004, 29:1069-1076. RCTs reference List (for web publication only).

74. Kennedy S, Baxter GD, Kerr DP, Bradbury I, Park J, McDonough SM: Acupuncture for acute non-specific low back pain: a pilot randomised non-penetrating sham controlled trial. Compl Ther Med 2008, 16:139-146. RCTs reference List (for web publication only).

75. Kerr DP, Walsh DM, Baxter D: Acupuncture in the management of chronic low back pain: a blinded randomized controlled trial. Clin J Pain 2003, 19:364-370. RCTs reference List (for web publication only).

76. Kovacs F, Abraira V, Santos S, Díaz E, Gestoso M, Muriel A, del Real MT G, Mufraggi N, Noguera J, Zamora J, Spanish Back Pain Research Network: A comparison of two short education programs for improving low back pain-related disability in the elderly: a cluster randomized controlled trial. Spine 2007, 32:1053-1059. RCTs reference List (for web publication only).

77. Kuukkanen T, Mälkiä E: Effects of a three-month therapeutic exercise programme on flexibility in subjects with low back pain. Physio Res Inter 2000, 5:46-61. RCTs reference List (for web publication only).

78. Leclaire R, Esdaile JM, Suissa S, Rossignol M, Proulx R, Dupuis M: Back school in a first episode of compensated acute low back pain: a clinical trial to assess efficacy and prevent relapse. Arch Phys Med Rehab 1996, 77:673-679. RCTs reference List (for web publication only).

79. Lindström I, Ohlund C, Nachemson A: Physical performance, pain, pain behavior and subjective disability in patients with subacute low back pain. Scand J Rehab Med 1995, 27:153-160. RCTs reference List (for web publication only).

80. Linton SJ, Andersson T: Can chronic disability be prevented? A randomised trial of a cognitive-behavior intervention and two forms of information for patients with spinal pain. Spine 2000, 25:2825-2831. RCTs reference List (for web publication only).

81. Luijsterburg PA, Verhagen AP, Ostelo RW, van den Hoogen HJ, Peul WC, Avezaat CJ, Koes BW: Physical therapy plus general practitioners' care versus general practitioners' care alone for sciatica: a randomised clinical trial with a 12-month follow-up. Eur Spine J 2008, 17:509-517. RCTs reference List (for web publication only).

82. Machado LA, Azevedo DC, Capanema MB, Neto TN, Cerceau DM: Clientcentered therapy vs exercise therapy for chronic low back pain: a pilot randomized controlled trial in Brazil. Pain Med 2007, 8:251-258. RCTs reference List (for web publication only).

83. Mannion AF, Müntener M, Taimela S, Dvorak J: A randomized clinical trial of three active therapies for chronic low back pain. Spine 1999, 24:2435-2448. $\mathrm{RCTs}$ reference List (for web publication only).

84. Mannion AF, Müntener M, Taimela S, Dvorak J: Comparison of three active therapies for chronic low back pain: results of a randomized clinical trial with one-year follow-up. Rheumatology 2001, 40:772-778. RCTs reference List (for web publication only).

85. Maul I, Läubli T, Oliveri M, Krueger H: Long-term effects of supervised physical training in secondary prevention of low back pain. Eur Spine J 2005, 14:599-611. RCTs reference List (for web publication only).

86. Mehling WE, Hamel KA, Acree M, Byl N, Hecht FM: Randomized, controlled trial of breath therapy for patients with chronic low-back pain. Alt Ther Health Med 2005, 11:44-52. RCTs reference List (for web publication only).

87. Moseley L: Combined physiotherapy and education is efficacious for chronic low back pain. Aust J Physio 2002, 48:297-302. RCTs reference List (for web publication only).

88. Niemistö L, Lahtinen ST, Rissanen P, Lindgren KA, Sarna S, Hurri H: A randomized trial of combined manipulation, stabilizing exercises, and physician consultation compared to physician consultation alone for chronic low back pain. Spine 2003, 28:2185-2191. RCTs reference List (for web publication only).

89. Niemistö L, Rissanen P, Sarna S, Lahtinen ST, Lindgren KA, Hurri H: Cost-effectiveness of combined manipulation, stabilizing exercises, and 
physician consultation compared to physician consultation alone for chronic low back pain: a prospective randomized trial with 2-year follow-up. Spine 2005, 30:1109-1115. RCTs reference List (for web publication only).

90. Nordeman L, Nilsson B, Möller M, Gunnarsson R: Early access to physical therapy treatment for subacute low back pain in primary health care: a prospective randomized clinical trial. Clin J Pain 2006, 22:505-511. RCTs reference List (for web publication only).

91. Paatelma M, Kilpikoski S, Simonen R, Heinonen A, Alen M, Videman T: Orthopaedic manual therapy, McKenzie method or advice only for low back pain in working adults: a randomized controlled trial with one year follow-up. J Rehab Med 2008, 40:858-863. RCTs reference List (for web publication only).

92. Peloso PM, Fortin L, Beaulieu A, Kamin M, Rosenthal N: Analgesic efficacy and safety of tramadol/ acetaminophen combination tablets (Ultracet) in treatment of chronic low back pain: a multicenter, outpatient, randomized, double blind, placebo controlled trial. J Rheum 2004, 31:2454-2463. RCTs reference List (for web publication only).

93. Rantonen J, Luoto S, Vehtari A, Hupli M, Karppinen J, Malmivaara A, Taimela $S$ : The effectiveness of two active interventions compared to self-care advice in employees with non-acute low back symptoms: a randomised, controlled trial with a 4-year follow-up in the occupational health setting. Occu Enviro Med 2012, 69:12-20. RCTs reference List (for web publication only).

94. Rasmussen BE, Nilsson WL, Arvidsson I: Stabilizing training compared with manual treatment in sub-acute and chronic low-back pain. Man Ther 2003, 8:233-241. RCTs reference List (for web publication only).

95. Rasmussen BE, Ang B, Arvidsson I, Nilsson WL: Graded exercise for recurrent low-back pain: a randomized, controlled trial with 6-, 12-, and 36-month follow-ups. Spine 2009, 34:221-228. RCTs reference List (for web publication only).

96. Rittweger J, Just K, Kautzsch K, Reeg P, Felsenberg D: Treatment of chronic lower back pain with lumbar extension and whole-body vibration exercise: a randomized controlled trial. Spine 2002, 27:1829-1834. RCTs reference List (for web publication only).

97. Ritvanen T, Zaproudina N, Nissen M, Leinonen V, Hänninen O: Dynamic Surface electromyographic responses in chronic low back pain treated by traditional bone setting and conventional physical therapy. J Manip Physio Ther 2007, 30:31-37. RCTs reference List (for web publication only).

98. Rossignol M, Abenhaim L, Séguin P, Neveu A, Collet JP, Ducruet T, Shapiro $\mathrm{S}$ : Coordination of primary health care for back pain. A randomised controlled trial. Spine 2000, 25:251-258. RCTs reference List (for web publication only).

99. Sahin N, Albayrak I, Durmus B, Ugurlu H: Effectiveness of back school for treatment of pain and functional disability in patients with chronic low back pain: a randomized controlled trial. J Rehab Med 2011, 43:224-229. RCTs reference List (for web publication only).

100. Soukup MG, Glomsröd B, Lönn JH, Bö K, Larsen S: The effect of a Mensendieck exercise program as secondary prophylaxis for recurrent low back pain. A randomized, controlled trial with 12-month follow-up. Spine 1999, 24:1585-1591. RCTs reference List (for web publication only).

101. Staal JB, Hlobil H, Twisk JW, Smid T, Köke AJ, van Mechelen W: Graded activity for low back pain in occupational health care: a randomized, controlled trial. Ann Int Med 2004, 140:77-84. RCTs reference List (for web publication only).

102. Hlobil H, Staal JB, Twisk J, Köke A, Ariëns G, Smid T, van Mechelen W: The effects of a graded activity intervention for low back pain in occupational health on sick leave, functional status and pain: 12-month results of a randomized controlled trial. J Occup Rehab 2005, 15:569-580 $\mathrm{RCTs}$ reference List (for web publication only).

103. Torstensen TA, Ljunggren AE, Meen HD, Odland E, Mowinckel P, Geijerstam $S$ : Efficiency and costs of medical exercise therapy, conventional physiotherapy, and self-exercise in patients with chronic low back pain. A pragmatic, randomized, single-blinded, controlled trial with 1-year follow-up. Spine 1998, 23:2616-2624. RCTs reference List (for web publication only).

104. Tsui ML, Cheing GL: The effectiveness of electroacupuncture versus electrical heat acupuncture in the management of chronic low-back pain. J Alt Comp Med 2004, 10:803-809. RCTs reference List (for web publication only).

105. Turner JA, Jensen MP: Efficacy of cognitive therapy for chronic low back pain. Pain 1993, 52:169-177. RCTs reference List (for web publication only).
106. Unsgaard TM, Fladmark AM, Salvesen $\varnothing$, Vasseljen O: Motor control exercises, sling exercises, and general exercises for patients with chronic low back pain: a randomized controlled trial with 1-year follow-up. Phys Ther 2010, 90:1426-1440. RCTs reference List (for web publication only).

107. van der Roer N, van Tulder M, Barendse J, Knol D, van Mechelen W, de Vet $\mathrm{H}$ : Intensive group training protocol versus guideline physiotherapy for patients with chronic low back pain: a randomised controlled trial. Eur Spine J 2008, 17:1193-1200. RCTs reference List (for web publication only).

108. Wand BM, Bird C, McAuley JH, Doré CJ, MacDowell M, De-Souza LH: Early intervention for the management of acute low back pain: a single-blind randomized controlled trial of biopsychosocial education, manual therapy, and exercise. Spine 2004, 29:2350-2356. RCTs reference List (for web publication only).

109. Werners R, Pynsent PB, Bulstrode CJ: Randomized trial comparing interferential therapy with motorized lumbar traction and massage in the management of low back pain in a primary care setting. Spine 1999, 24:1579-1584. RCTs reference List (for web publication only).

110. Yelland MJ, Glasziou PP, Bogduk N, Schluter PJ, McKernon M: Prolotherapy injections, saline injections, and exercises for chronic low-back pain: a randomized trial. Spine 2004, 29:9-16. RCTs reference List (for web publication only).

111. Gartlehner G, Hansenb RA, Nissmanc D, Lohrd KN, Carey TS: A simple and valid tool distinguished efficacy from effectiveness studies. J Clin Epidemiol 2006, 59:1040-1048.

112. Thorpe KE, Zwarenstein M, Oxman AD, Treweek S, Furberg CD, Altman DG, Tunis S, Bergel E, Harvey I, Magid DJ, Chalkidou K: A pragmaticexplanatory continuum indicator summary (PRECIS): a tool to help trial designers. J Clin Epidemiol 2009, 62:464-475

113. Shrier I, Boivin JF, Steele RJ, Platt RW, Furlan A, Kakuma R, Brophy J, Rossignol M: Should meta-analyses of interventions include observational studies in addition to randomized controlled trials? A critical examination of underlying principles. Am J Epidem 2007, 166:1203-1209.

114. Tzoulaki I, Siontis KCM, loannidis JPA: Prognostic effect size of cardiovascular biomarkers in datasets from observational studies versus randomised trials: meta-epidemiology study. BMJ 2011, 343:d6829.

115. Schouten BC, Meeuwesen $L$, Harmsen HAM: The impact of an intervention in intercultural communication on doctor-patient interaction in The Netherlands. Patient Counsell Health Educ 2005, 58:288-295.

116. Schouten BC, Meeuwesen L, Tromp F, Harmsen HAM: Cultural diversity in patient participation: The influence of patients' characteristics and doctors' communicative behaviour. Patient Counsell Health Educ 2007, 67:214-223.

117. Smith SK, Dixon A, Trevena L, Nutbeam D, McCaffery KJ: Exploring patient involvement in healthcare decision making across different education and functional health literacy groups. Soc Sci Med 2009, 69:1805-1812.

118. Shaw A, Ibrahim S, Reid F, Ussher M, Rowlands G: Patients' perspectives of the doctor-patient relationship and information giving across a range of literacy levels. Patient Counsell Health Educ 2009, 75:114-120.

119. Colloca L, Lopiano L, Lanotte M, Benedetti F: Overt versus covert treatment for pain, anxiety, and Parkinson's disease. Lancet Neurol 2004, 3:679-684.

\section{doi:10.1186/1471-2474-15-68}

Cite this article as: Artus et al.: The clinical course of low back pain: a meta-analysis comparing outcomes in randomised clinical trials (RCTs) and observational studies. BMC Musculoskeletal Disorders 2014 15:68. 